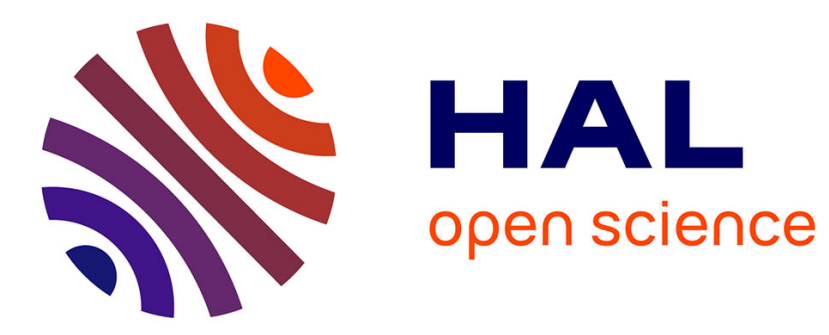

\title{
Enregistrement thermomagnétique dans les couches minces de manganèse-bismuth et applications
}

\author{
J.M. Rouchon, M. Vergnolle
}

\section{To cite this version:}

J.M. Rouchon, M. Vergnolle. Enregistrement thermomagnétique dans les couches minces de manganèse-bismuth et applications. Revue de Physique Appliquée, 1974, 9 (3), pp.557-564. 10.1051/rphysap:0197400903055700 . jpa-00243818

\section{HAL Id: jpa-00243818 https://hal.science/jpa-00243818}

Submitted on 1 Jan 1974

HAL is a multi-disciplinary open access archive for the deposit and dissemination of scientific research documents, whether they are published or not. The documents may come from teaching and research institutions in France or abroad, or from public or private research centers.
L'archive ouverte pluridisciplinaire HAL, est destinée au dépôt et à la diffusion de documents scientifiques de niveau recherche, publiés ou non, émanant des établissements d'enseignement et de recherche français ou étrangers, des laboratoires publics ou privés. 


\title{
ENREGISTREMENT THERMOMAGNÉTIQUE DANS LES COUCHES MINCES DE MANGANẼSE-BISMUTH ET APPLICATIONS (*)
}

\author{
J. M. ROUCHON et M. VERGNOLLE \\ THOMSON-CSF, Laboratoire Central de Recherches \\ Domaine de Corbeville, 91401 Orsay, France
}

(Reçu le 5 décembre 1973, révisé le 5 janvier 1974)

\begin{abstract}
Résumé. - Cet article décrit les recherches expérimentales sur l'enregistrement thermomagnétique dans les couches minces de MnBi. Des expériences de cycles répétés (inscription-effacement) ont été faites avec la phase normale et la phase trempée de ce matériau. Ces expériences effectuées en un point de $1 \mu \mathrm{m}$ de diamètre avec des largeurs d'impulsions voisines de $1 \mu$ s ont permis de définir les conditions d'inscription qui évitent tout dommage dû aux cycles répétés (environ 106). Ces résultats ont été utilisés pour l'enregistrement holographique. Dans ce cas, nous avons étudié la répartition spatiale de l'aimantation induite par l'analyse des propriétés de la lumière diffractée. En conclusion, la densité d'énergie nécessaire est $50 \mathrm{~mJ} / \mathrm{cm}^{2}$ et la capacité de stockage dans les deux processus d'enregistrement digital ou holographique est typiquement $10^{7} \mathrm{bits} / \mathrm{cm}^{2}$.
\end{abstract}

Abstract. - This paper describes experimental investigations of the thermomagnetic recording on $\mathrm{MnBi}$ thin films. Repeated cycling (writing-erasing) experiments have been performed in the normal phase and in the quenched phase of this material. These experiments on a $1 \mu \mathrm{m}$ diameter spot with about one microsecond laser pulse duration have facilitated the determination of the writing conditions which avoid damage due to repeated cycles (about 106). With these results we have performed holographic recording. In this case we have studied the spatial distribution of the induced magnetization by measuring the properties of the diffracted light beam. It has been concluded that the required light energy density is $50 \mathrm{~mJ} / \mathrm{cm}^{2}$ and the storage capacity in the digital and holographic processes is typically $10^{7} \mathrm{bits} / \mathrm{cm}^{2}$.

1. Introduction. - Les besoins croissants en stockage d'informations et archivages de données imposent d'étudier et de développer des mémoires de masses, c'est-à-dire des mémoires associant de grandes capacités à des débits écriture-lecture élevés. Les effets de mémoire caractéristiques des ferromagnétiques ont été largement utilisés jusqu'à présent, essentiellement pour la non-volatilité de l'information enregistrée et l'absence de dépense d'énergie pour la conserver. Quel que soit le mode d'utilisation de ces effets (matrices de tores, bandes et disques magnétiques) capacité de stockage et temps d'accès s'avèrent limités ; c'est en particulier pour ces raisons que d'importantes recherches sont faites sur les mémoires à semi-conducteur.

Une autre solution au problème posé consisterait à associer les techniques de l'optique qui permettent le transfert d'images, c'est-à-dire le transfert en parallèle de grandes quantités d'informations $\left(10^{4}\right.$ à $10^{5}$ bits) aux techniques de l'enregistrement magnétique. Il serait alors possible de profiter à la fois des avantages de l'accès aléatoire rapide et de la forte densité de

(*) Recherches supportées en partie par le C. R. I. et la D. R. M. E. stockage propres aux méthodes optiques et de ceux du stockage magnétique. Cette solution nécessite un milieu dans lequel il y ait interaction des propriétés magnétiques et optiques. Cette interaction, dite effet photomagnétique, n'existe à température ambiante avec une amplitude utilisable dans aucun matériau connu à ce jour. Il existe cependant l'effet thermomagnétique, qui permet par absorption d'un rayonnement lumineux dans un matériau magnétique de l'échauffer localement et de créer des états magnétiques distincts par passage au-dessus du point de Curie. Un matériau dans lequel l'effet Kerr ou l'effet Faraday serait particulièrement élevé permettrait donc l'observation optique de l'effet induit et pourrait être utilisé pour l'enregistrement et la lecture optique d'informations.

Les couches minces du composé manganèse-bismuth satisfont aux conditions précédentes; le présent article reporte les résultats obtenus lors de l'étude détaillée des processus d'enregistrement et de lecture optiques, ainsi que de l'effacement. Nous avons procédé tout d'abord à des enregistrements ponctuels, puis à des enregistrements holographiques : ceux-ci permettent en effet le transfert en parallèle de toute la 
quantité d'information contenue dans une " page » et réduisent les difficultés de positionnement mécanique à la lecture ainsi que l'influence des inhomogénéités de la couche.

2. Rappel des propriétés du MnBi et des processus d'inscription, lecture, effacement. - Le composé Manganèse-Bismuth $(\mathrm{MnBi})$ est ferromagnétique à la température ambiante et possède une structure hexagonale du type NiAs. Il présente deux formes cristallographiques : une forme stable $\varphi_{\mathrm{s}}$ obtenue par refroidissement lent de l'alliage depuis $400^{\circ} \mathrm{C}$ et une forme métastable $\varphi_{\mathrm{m}}$ obtenue par trempe de l'alliage depuis cette même température [1]. Entre $340^{\circ} \mathrm{C}$ et $360^{\circ} \mathrm{C}$ le composé $\mathrm{MnBi}$ présente une transition de phase; la phase haute température obtenue au delà de ces températures n'est plus ferromagnétique. Cette transition de phase se produit avec hystérésis thermique qui dépend des vitesses de chauffage et de refroidissement $[2,3]$. A température ambiante $\varphi_{\mathrm{m}}$ tend à évoluer vers $\varphi_{\mathrm{s}}$ et la cinétique de cette transformation a été étudiée par diffraction $\mathrm{X}$. Ainsi au voisinage de $30^{\circ} \mathrm{C}$, une lame mince de Manganèse-Bismuth initialement en phase $\varphi_{\mathrm{m}}$ présente après 10 jours $20 \%$ de phase $\varphi_{\mathrm{s}}$. Ceci explique que les premières expériences ont été faites avec MnBi en phase $\varphi_{\mathrm{s}}$, l'utilisation de la phase $\varphi_{\mathrm{m}}$ nécessitant de résoudre le problème de sa stabilisation.

2.1 INSCRIPTION, LECTURE, EFFACEMENT. - Grâce à la présence d'un champ d'anisotropie très élevé, il est possible d'obtenir des couches minces de ManganèseBismuth, sur substrat de verre, avec une direction d'aimantation perpendiculaire au plan de la couche.

L'inscription thermomagnétique en un point d'une lame $\mathrm{MnBi}$ en phase normale $\varphi_{\mathrm{s}}$ est obtenue en portant ce point («bit ») à une température supérieure à $360^{\circ} \mathrm{C}$. Un faisceau laser focalisé sur la couche permet d'obtenir les densités d'énergie suffisantes pour induire cette élévation de température. L'aimantation de la zone chauffée disparaît au passage de la température de Curie ; lors du refroidissement, elle prend le sens du champ démagnétisant $H_{\mathrm{d}}$ dû aux zones avoisinantes (Fig. 1). Ce champ est de sens opposé

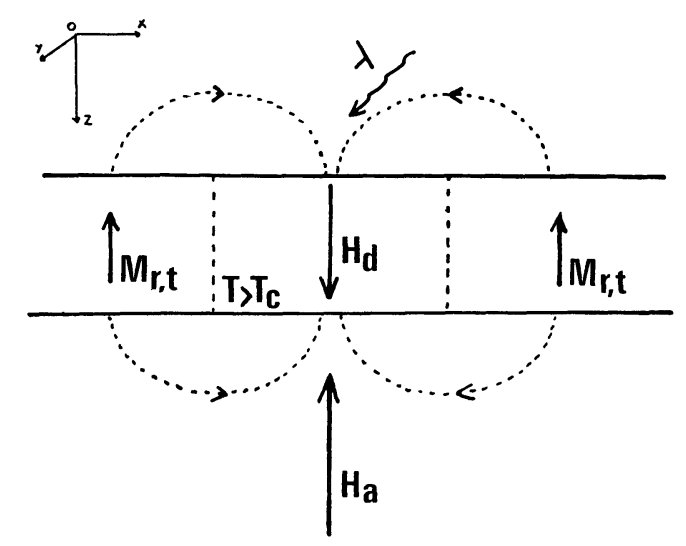

Fig. 1. - Inscription et effacement thermomagnétique $\left(H_{\mathrm{d}}\right.$ : champ démagnétisant d'écriture, $H_{\mathrm{a}}:$ champ appliqué à l'effacement). à celui de l'aimantation initiale et induit par conséquent une variation locale de l'aimantation dans la zone éclairée. Cette zone constitue un bit d'information qui peut être détecté soit par effet Kerr (réflexion), soit par effet Faraday (transmission). Dans le cas de l'effet Faraday, le plan de polarisation d'un faisceau de lecture polarisé linéairement tourne après transmission d'un angle $\theta_{\mathrm{F}}$ lié au sens de l'aimantation, à l'épaisseur traversée et à la valeur de l'aimantation. En se plaçant derrière un analyseur faisant l'angle $\left(\frac{\pi}{2}-\theta_{0}\right)$ avec la direction de polarisation du faisceau de lecture incident, l'intensité transmise est définie par la relation :

$$
I=K I_{0} \mathrm{e}^{-\alpha s} \sin ^{2}\left(\theta_{0}+\varepsilon \theta_{\mathrm{F}}\right) .
$$

Avec :

$K$ constante liée au coefficient de réflexion sur la couche,

$\alpha$ coefficient d'absorption de la couche,

$I_{0}$ intensité incidente,

$s$ épaisseur de la couche,

$\theta_{\mathrm{F}}=F \times s$ avec $F$ rotation Faraday de l'ordre de $10^{6}$ degrés $/ \mathrm{cm}$.

$\varepsilon=+1$ si l'aimantation est $+M_{s}$ et $\varepsilon=-1$ si l'aimantation est $-M_{s}$.

Dans le cas où l'analyseur est croisé avec l'une des 2 directions correspondant à $+M_{s}$ ou $-M_{s}$, la différence d'intensité transmise entre 2 zones d'aimantations antiparallèles est :

$$
\Delta I=K I_{0} \mathrm{e}^{-\alpha S} \sin ^{2} 2 \theta_{\mathrm{F}} .
$$

Le maximum de contraste est donc fonction de l'épaisseur de la couche $\mathrm{MnBi}$. Pour des couches telles que $\alpha \simeq 4 \times 10^{5} \mathrm{~cm}^{-1}$ (cas typique de $\mathrm{MnBi}$ ), les épaisseurs optimales à utiliser sont de l'ordre de $50 \mathrm{~nm}$.

L'effacement de l'information inscrite se fait par un processus identique à celui de l'écriture, à la différence que l'on ajoute un champ $H_{\mathrm{a}}$ (typiquement de l'ordre de $1500 \mathrm{Oe}$ ) opposé au champ démagnétisant $H_{\mathrm{d}}$, de telle sorte que $\left(H_{\mathrm{a}}-H_{\mathrm{d}}\right)>0$. Ainsi, pendant le refroidissement, l'aimantation reprend son sens initial, ce qui conduit à l'effacement de l'information enregistrée.

Pour la phase métastable $\varphi_{\mathrm{m}}$, qui présente une température de Curie plus faible $\left(160^{\circ} \mathrm{C}\right)$, l'énergie nécessaire à l'inscription est inférieure à celle requise par la phase $\varphi_{\mathrm{s}}$. Cette phase a toutefois une aimantation à saturation inférieure à celle de $\varphi_{\mathrm{s}}$. Le champ $H_{\mathrm{a}}$ nécessaire à l'effacement est donc plus faible. La rotation Faraday étant proportionnelle à l'aimantation à saturation, nous aurons en outre à la lecture une diminution du rapport signal sur bruit.

Quel que soit le mode d'enregistrement choisi (ponctuel ou holographique) l'information enregistrée est liée à la répartition spatiale de la température obtenue lors de l'inscription. Nous allons rappeler brièvement 
les principaux résultats sur le calcul de cette température.

2.2 RÉPARTITION THERMIQUE AU COURS DE L'INSCRIPTION. - Le calcul de la température atteinte par une couche mince de $\mathrm{MnBi}$ se fait par intégration des équations de propagation de la chaleur. Dans le cas général d'une couche mince d'épaisseur $s$ (conductivité thermique $K_{1}$, diffusivité thermique $k_{1}$ ) déposée sur un substrat d'épaisseur $S\left(K_{2}, k_{2}\right)$ la température atteinte lors de l'inscription d'un point par un faisceau laser gaussien ne peut être exprimée de façon explicite. Si on considère le cas plus simple d'un milieu d'absorption $\alpha$ déposé sur un substrat transparent, la température atteinte au centre du point peut se mettre sous la forme [4]

$$
\begin{array}{ll}
\theta(0, t)=\frac{Q_{\mathrm{T}}}{4 \pi K_{1} s} \log \left(1+\frac{4 k_{1} t}{a^{2}}\right) & t<T \\
\theta(0, t)=\frac{Q_{\mathrm{T}}}{4 \pi K_{1} s} \log \left(\frac{a^{2}+4 k_{1} t}{a^{2}+4 k_{1}(t-T)}\right) & t>T
\end{array}
$$

$T$ étant le temps d'exposition,

$a$ rayon du faisceau laser à $1 / e$ dont l'intensité lumineuse varie suivant la loi

$$
I=I_{0} \mathrm{e}^{-\frac{x^{2}+y^{2}}{a^{2}}},
$$

$Q_{\mathrm{T}}$ quantité de chaleur totale fournie

$$
Q_{\mathrm{T}}=I_{0} \pi a^{2}\left(1-\mathrm{e}^{-\alpha s}\right) T \text {. }
$$

Compte tenu des densités d'informations et des débits souhaités, à l'enregistrement comme à l'effacement, les inscriptions ponctuelles doivent être faites sur des surfaces de l'ordre de $1 \mu \mathrm{m}^{2}$ et en des temps inférieurs ou égaux à $1 \mu \mathrm{s}$. D'après les relations précédentes, les puissances lumineuses requises sont de quelques milliwatts et les vitesses de chauffage et de refroidissement sont de plusieurs centaines de degrés par microseconde. Or, aucune étude de changement de phase n'a été faite à de telles vitesses. De plus, se pose le problème de la fatigue du matériau lors de chauffages répétés au même point. Ce sont les deux points par lesquels ont débuté nos études.

Dans le cas de l'enregistrement holographique (Fig. 2), le calcul de la température atteinte est un peu plus complexe puisque l'on a un éclairement périodique suivant la direction $o x$ de la forme

$$
I_{0}\left(1+\cos \frac{2 \pi x}{d}\right)
$$

pendant le temps $T$ d'exposition.

En première approximation, pour une couche isolée et sans tenir compte de la répartition gaussienne de l'intensité dans les 2 ondes qui interfèrent, R. S. Mezrich [5] a montré que la température pouvait se mettre sous la forme pour $t<T$ :

$$
\theta(x, t)=\frac{k I_{0} t}{K s}\left(1+m(t) \cos \frac{2 \pi x}{d}\right)
$$

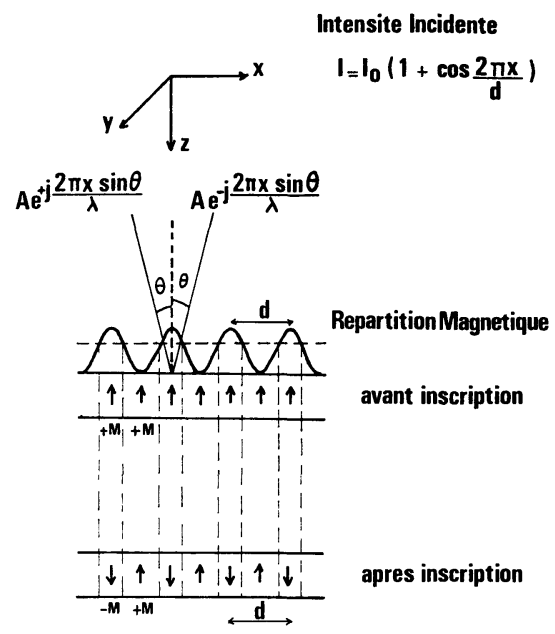

Fig. 2. - Inscription thermomagnétique d'un hologramme sur $\mathrm{MnBi}$.

avec

$$
m(t)=\frac{d^{2}}{4 \pi^{2} k t}\left(1-\mathrm{e}^{\frac{-4 \pi^{2} k t}{d^{2}}}\right)
$$

et pour $t>T$

$$
\begin{aligned}
\theta(x, t)= & \frac{k I_{0} T}{K s}+\frac{I_{0} d^{2}}{4 \pi^{2} K s} \times \\
& \times \mathrm{e}^{\frac{-4 \pi^{2} k(t-T)}{d^{2}}}\left(1-\mathrm{e}^{\frac{-4 \pi^{2} k T}{d^{2}}}\right) \cos \frac{2 \pi x}{d} .
\end{aligned}
$$

Ce système d'équations permet de définir les conditions de formation d'un hologramme sur une couche mince de $\mathrm{MnBi}$.

Compte tenu des constantes thermiques du $\mathrm{MnBi}$ ( $k$ de l'ordre de $0,05 \mathrm{~cm}^{2} / \mathrm{s}$ ) [6], pour un interfrange $d$ de $1 \mu \mathrm{m}$, le terme $d^{2} / 4 \pi^{2} k$ est voisin de $5 \mathrm{~ns}$. Cette faible constante de temps impose d'utiliser pour l'inscription thermomagnétique d'un hologramme des impulsions de chauffage de faible durée (quelques dizaines de nanosecondes) afin de conserver un facteur de modulation $m(t)$ voisin de 1 . D'autre part, l'équation (7) montre que pendant l'enregistrement, la couche atteint une température moyenne $\theta_{0}=k I_{0} T / K s$.

$\theta_{0}$ doit rester inférieure à la température de Curie sinon le sens de l'aimantation serait inversé dans toute la zone chauffée ; il n'y aurait donc pas enregistrement d'hologramme. Signalons enfin que dans tous les cas, la température maximum atteinte doit rester inférieure à $450^{\circ} \mathrm{C}$, température à laquelle se produit la destruction de la couche.

Dans le but d'évaluer les caractéristiques du $\mathrm{MnBi}$ en tant que support optique d'information nous avons donc été conduits à deux séries d'expériences. Lors d'enregistrements de points, nous avons effectué les tests de fatigue du matériau ainsi que les tests permettant de définir la densité maximum propre à ce mode d'enregistrement ; partant de ces résultats, nous avons ensuite procédé aux essais d'enregistrement 
d'informations en parallèle par utilisation des techniques holographiques.

3. Etude de l'évolution des caractéristiques du matériau lors de cycles répétés inscription-effacement en un même point. - Pour toute utilisation informatique il est nécessaire de savoir si les caractéristiques d'un point mémoire sont dépendantes ou non des antécédents de ce point ou de son voisinage. Nous avons suivi l'évolution de ces caractéristiques lors de cycles répétés en analysant les signaux de lecture en effet Faraday. A la lecture, le faisceau utilisé est le même que celui ayant servi à l'inscription, mais il est atténué dans un rapport 5 de façon à ne pas perturber la couche.

La figure 3 donne le schéma d'un cycle de test ; les écarts $X_{1}, X_{2}, X_{3}$ entre les 4 phases de ce cycle (écriture, lecture après écriture, effacement, lecture après effacement) sont réglables à partir d'une centaine de nanosecondes, ainsi que les largeurs des impulsions lumineuses utilisées. La fréquence de répétition de ces cycles peut atteindre $10 \mathrm{MHz}$.

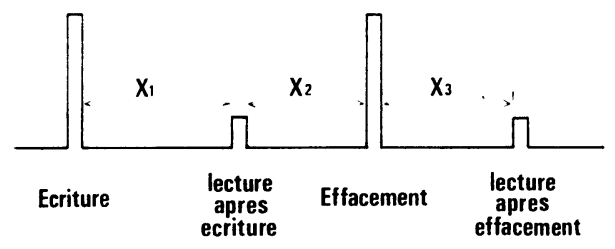

Fig. 3. - Schéma d'un cycle de test.

3.1 Dispositif EXPÉRIMENTAL. - L'ensemble des éléments utilisés pour ces expériences est constitué principalement (Fig. 4) par :

- un laser Argon : $P_{\max }=1 \mathrm{~W}$ à $488 \mathrm{~nm}$,

- un modulateur acousto-optique : il délivre les impulsions lumineuses pour l'inscription et l'effacement et sa fréquence maximum d'utilisation est de $25 \mathrm{MHz}$,

- un déflecteur XY acousto-optique : il permet l'adressage de $100 \times 100$ points distincts sur la couche,

- un ensemble optique pour la focalisation du faisceau laser : le diamètre du faisceau au niveau de la lame $\mathrm{MnBi}$ est de $1,8 \mu \mathrm{m}$ (à $1 / e^{2}$ ),

- une bobine utilisée à l'effacement qui produit des champs pulsés d'amplitude maximum 1800 Oe au niveau de la lame,

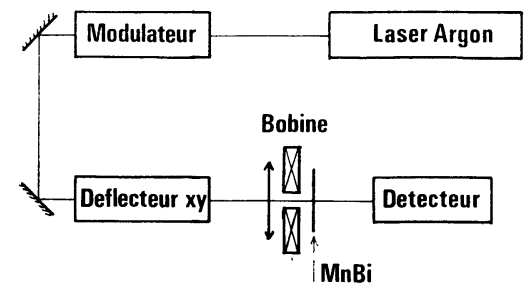

Fig. 4. - Schéma du banc utilisé pour effectuer les cycles inscription-effacement.
- la couche mince de $\mathrm{MnBi}$ déposée sur substrat de verre,

- un ensemble de détection optique de l'état magnétique obtenu après inscription et effacement. Les signaux de lecture sont traités à l'aide d'un analyseur multicanaux qui permet d'obtenir leur spectre d'amplitude après écriture et après effacement.

L'électronique de commande destinée à enchaîner les fonctions élémentaires formant une séquence de test est constituée par des générateurs modulaires commandés par une logique dont le synchronisme est réalisé à partir d'un oscillateur à quartz.

Toutes les expériences ont été effectuées à température ambiante; la première série de tests a été faite sur une lame en phase stable.

3.2 CYCLES RÉPÉTÉS ÉCRITURE-EFFACEMENT SUR UNE LAME EN PHASE STABle. - La mise en évidence de l'influence de $N$ chauffages successifs en un même point sur les caractéristiques du matériau a été effectuée selon deux procédés : d'une part, en analysant les signaux de lectures après écriture $\left(I_{1}\right)$ et après effacement $\left(I_{2}\right)$,

d'autre part, en observant au microscope, par effet Faraday, l'aspect du point qui a subi les cycles répétés.

Soient $P_{0}$ la puissance seuil nécessaire à l'inscription d'un point en un temps $T_{0}, P_{\max }$ la puissance maximum à utiliser pour ne pas dépasser la température de destruction en une seule impulsion.

Les puissances seuils obtenues au cours de nos expériences avec différentes largeurs d'impulsion sont données dans le tableau suivant :

\begin{tabular}{|c|c|}
\hline$T_{0} \mu \mathrm{s}$ & $P_{0}$ \\
\hline 0,2 & $11 \mathrm{~mW}$ \\
\hline 0,5 & $6 \mathrm{~mW}$ \\
\hline 1 & $4,8 \mathrm{~mW}$ \\
\hline 3 & $4,2 \mathrm{~mW}$ \\
\hline
\end{tabular}

Quelle que soit la puissance $P\left(P_{\max }>P \geqslant P_{0}\right)$ utilisée à l'inscription lors des cycles répétés, nous constatons tout d'abord une diminution de l'écart entre les lectures $I_{1}$ et $I_{2}$. Cette diminution est d'autant plus rapide que la différence entre $P$ et $P_{0}$ est plus grande. La figure 5 donne un exemple de ce phénomène, observé au cours des 100 premiers cycles inscription-effacement. Notons qu'après cette diminution initiale de l'écart $\left(I_{1}-I_{2}\right)$, celui-ci se stabilise. Si on augmente ensuite le nombre $N$ de cycles jusqu'à $N$ égal $10^{6}, 2$ phénomènes peuvent se produire :

- Si $P_{0}<P<1,1 P_{0}$, l'écart $\left(I_{1}-I_{2}\right)$ reste constant, l'observation du point mémoire au microscope ne fait apparaître aucun signe de destruction.

- Si $P_{\max }>P>1,1 P_{0}$, les intensités $I_{1}$ et $I_{2}$ des 2 lectures augmentent progressivement avec les valeurs de $N$ et $P$; l'observation du point mémoire au microscope fait apparaître une destruction du matériau dans sa partie centrale qui ne présente alors plus d'effet Faraday ; la surface de la partie détruite dépend de $N$ et $P$. 


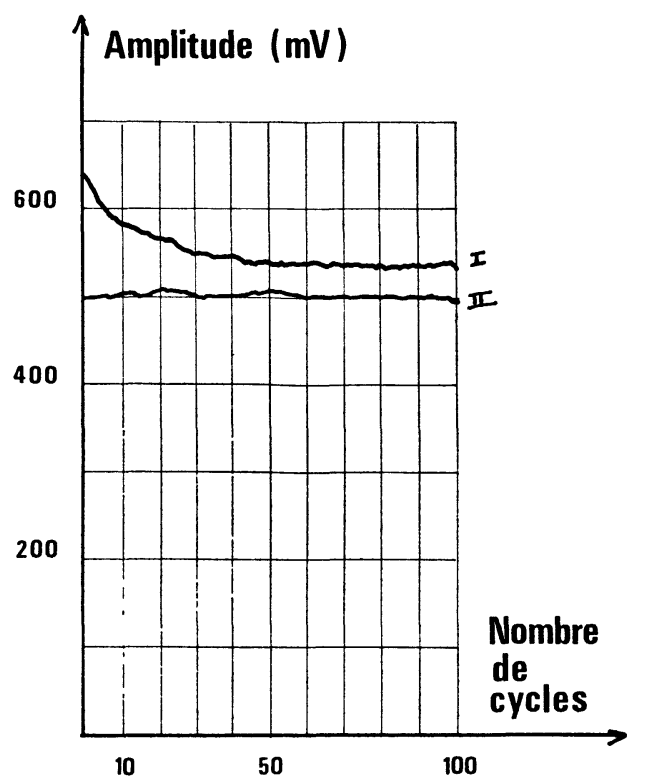

FIG. 5. - Amplitude des lectures après écriture (I) et après effacement (II) au cours de 100 cycles inscription-Effacement effectués avec des impulsions de largeur $3 \mu \mathrm{s}$ et puissance $4,75 \mathrm{~mW}$ ( $20 \%$ au-dessus du seuil).

Cette tolérance sur la variation de puissance $(10 \%$ au-dessus de $P_{0}$ ) est fonction de la fréquence des inscriptions. Pour des impulsions de $1 \mu \mathrm{s}$, la valeur indiquée est valable jusqu'à des fréquences de $20 \mathrm{kHz}$.

L'ensemble de ces résultats montre qu'au cours de cycles répétés, à puissance trop élevée, il est possible d'induire une élévation progressive de la température jusqu'à la température de destruction $T_{\mathrm{d}}$. En outre, même en se plaçant dans le domaine des puissances tolérables telles que la température de régime soit inférieure à $T_{\mathrm{d}}$, nous avons noté une diminution de l'écart $\left(I_{1}-I_{2}\right)$ au cours des premiers cycles inscription-effacement. Ce phénomène est d'autant plus rapide que la puissance utilisée à l'inscription est plus élevée. Il peut s'expliquer par une transition progressive de la phase $\varphi_{\mathrm{s}}$ à la phase $\varphi_{\mathrm{m}}$. En effet, lorsque l'écart $\left(I_{1}-I_{2}\right)$ est constant, nous trouvons au point où les

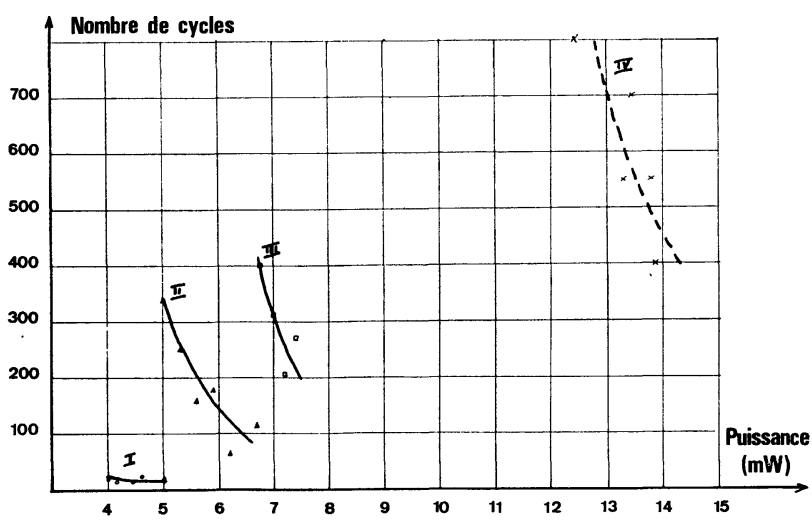

Fig. 6. - Nombre de cycles inscription-effacement au bout duquel il y a changement de phase au point de test en fonction de la puissance lumineuse envoyée sur la couche pour différentes largeurs d'impulsion (I : $3 \mu \mathrm{s}, \mathrm{II}: 1 \mu \mathrm{s}, \mathrm{III}: 0,5 \mu \mathrm{s}, \mathrm{IV}: 0,2 \mu \mathrm{s}$ ). cycles répétés ont été effectués les conditions d'écriture et d'effacement qui s'avèrent, comme nous le montrons dans la suite, caractéristiques d'une couche en phase trempée : puissance de l'impulsion de chauffage et champ d'effacement ont diminué respectivement dans un rapport 4 et 2 . La figure 6 donne, pour différentes largeurs d'impulsion, le nombre de cycles nécessaire pour obtenir ce changement de phase en fonction de la puissance utilisée.

Ces résultats sont à rapprocher de ceux obtenus par Di Chen et al. [7] qui ont observé lors de cycles répétés d'une part, la transformation de phase, d'autre part, la destruction du matériau pour certaines valeurs de puissance utilisée à l'inscription. Les tolérances sur la variation de puissance d'inscription sont plus sévères dans nos expériences (10\% au-dessus du seuil). Ceci est lié aux conditions expérimentales différentes : diamètre du faisceau laser d'inscription plus faible et fréquence de répétition des cycles plus élevée.

\section{3 CYCLES ÉCRITURE - EFFACEMENT SUR UNE LAME} EN PHASE TREMPÉE. - La température de Curie est dans ce cas égale à $160^{\circ} \mathrm{C}$, donc beaucoup plus éloignée de $T_{\mathrm{d}}$ que dans le cas de la phase $\varphi_{\mathrm{s}}$. Ainsi les marges de puissances tolérables à l'inscription sont beaucoup plus grandes. Sur la figure 7 nous avons représenté l'amplitude des lectures au cours de 100 cycles effectués avant et après $10^{6}$ inscriptions au même point. pour une puissance égale à 2 fois la puissance seuil $P_{0}$; le spectre d'ámplitude des lectures après écriture au cours des $10^{6}$ cycles écriture-lecture effectués à $200 \mathrm{kHz}$ est schématisé sur la courbe $\mathrm{C}$ de la figure 7 .

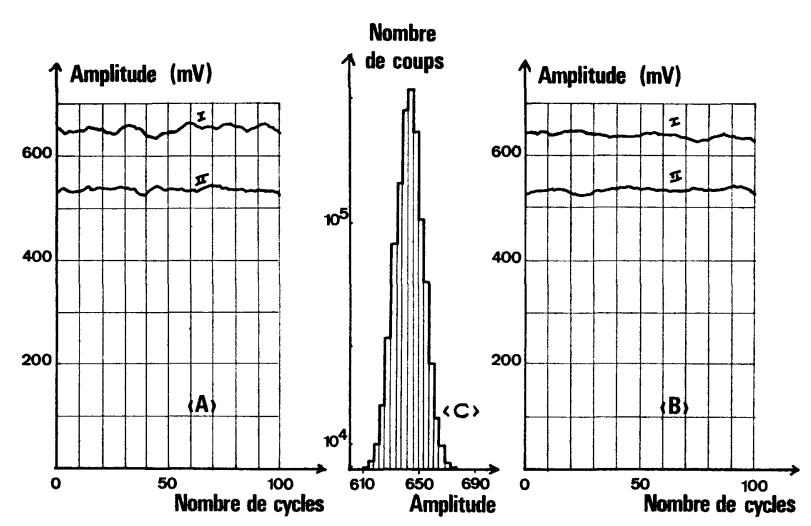

Fig. 7. - Amplitude des lectures après écriture (I) et après effacement (II) avant (A) et après (B) $10^{6}$ inscriptions au même point. Courbe $(C)$ : spectre d'amplitude des lectures après écriture au cours des $10^{6}$ cycles écriture-lecture effectués à $200 \mathrm{kHz}$ (impulsion $P=2,4 \mathrm{~mW}, T=1 \mu \mathrm{s}$ ).

Nous avons pu constater ainsi qu'avec des impulsions de $1 \mu \mathrm{s}$, le matériau conserve ses propriétés initiales après $10^{6}$ inscriptions effectuées au même point à une fréquence de $500 \mathrm{kHz}$ et une puissance égale à $4 P_{0}$.

Cependant si l'emploi de la phase $\varphi_{\mathrm{m}}$ est plus aisé lors de telles expériences, cette phase se transforme lentement en phase $\varphi_{\mathrm{s}}$, en l'absence de toute action 
extérieure. Elle ne procure donc pas le caractère requis de non-volatilité de l'information enregistrée. C'est pourquoi de nombreux laboratoires se sont intéressés à la stabilisation de cette phase. Cette stabilisation peut s'obtenir en modifiant les liaisons interatomiques, soit par substitutions ioniques, soit par ajouts d'atomes interstitiels dans le réseau $\mathrm{MnBi}$. L'utilisation du Titane [8] ou du Nickel [9] a permis une diminution de 1 à 3 ordres de grandeurs des vitesses de transformation.

3.4 Tests De Perturbations. - Après avoir défini les conditions dans lequelles le matériau conserve ses caractéristiques après $N$ cycles inscription-effacement, il est nécessaire de définir la densité de stockage d'informations que l'on peut atteindre. Par l'adressage d'un carré de $3 \times 3$ points il est possible de déterminer l'influence des 8 points périphériques sur le point central au cours de l'enregistrement et de l'effacement. L'espacement minimum mesuré pour qu'il n'y ait pas interaction est de $2,3 \mu \mathrm{m}$ pour des points de diamètre $1 \mu \mathrm{m}$. Cet espacement conduit à des densités de stockage voisines de $2 \times 10^{7}$ bits $/ \mathrm{cm}^{2}$. La figure 8 montre un exemple de points inscrits avec un tel espacement.

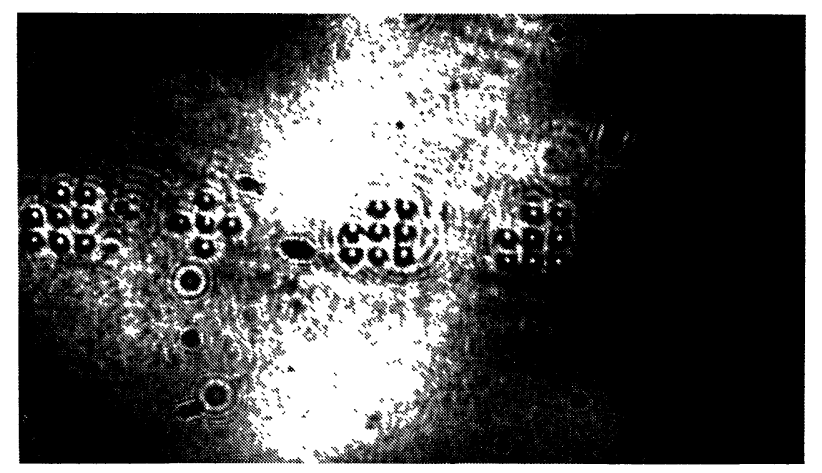

Fig. 8. - Visualisation au microscope en effet Faraday de bits inscrits sur MnBi lors des tests de perturbations $(G=900)$.

L'ensemble des caractéristiques relatives aux inscriptions ponctuelles successives étant maintenant connu, nous étudierons les possibilités d'utilisation de ces couches en tant que support d'enregistrement holographique.

4. Utilisation des couches minces de MnBi comme support d'enregistrement holographique. - Les études ont porté à la fois sur les sources lumineuses, les systèmes optiques capables de transférer la quantité d'informations contenues dans une page et sur l'aptitude des couches à l'enregistrement optique holographique.

D'après les résultats indiqués en 2.2 , la source lumineuse choisie est un laser Rubis déclenché capable de fournir en des temps de quelques dizaines de nanosecondes des énergies de l'ordre du millijoule.

4.1 Montage eXPérimental. - Le montage expérimental pour l'étude de la formation d'hologrammes sur $\mathrm{MnBi}$ est schématisé sur la figure 9 . Un système lame $1 / 2$ onde-Wollaston permet de faire varier les intensités relatives des 2 faisceaux. Placé sur le trajet du faisceau de référence, un miroir mobile permet de faire varier l'angle entre les 2 faisceaux de $10^{\circ}$ à $70^{\circ}$, la différence de chemin optique entre les 2 faisceaux restant compatible avec la longueur de cohérence du laser (*). Pour l'enregistrement de l'hologramme d'un plan-objet binaire nous avons choisi un montage de Fourier. Afin d'éviter sur la lame les fortes variations d'éclairement entre les faibles et les fortes fréquences spatiales qui se produisent dans le plan spectral, une translation de l'objectif $\mathrm{O}_{2}$ permet de placer la lame $\mathrm{MnBi}$ en dehors de ce plan. Dans le cas où l'objet est un peigne de Dirac de pas $p, l$ la distance de $\mathrm{O}_{2}$ à la lame $\mathrm{MnBi}, D$ la dimension de l'objet, l'énergie dans le plan spectral est localisée dans des pics de diffraction dont le pas est $p^{\prime}=\lambda l / p, \lambda$ longueur d'onde du laser d'inscription.

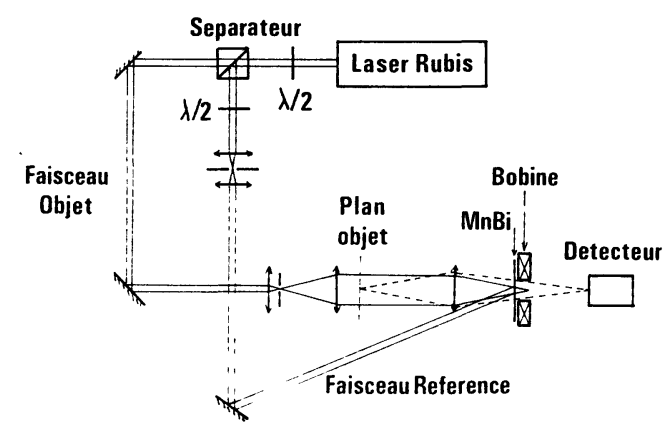

FIG. 9. - Schéma du banc d'inscription-effacement d'un hologramme sur MnBi.

Le spectre est continu lorsque la défocalisation $d$ vaut :

$$
d= \pm \frac{\lambda l^{2}}{D p}
$$

La défocalisation de la lame évitera donc les forts écarts de température dans son plan.

La bobine d'effacement donnant des champs variables jusqu'à 2000 Oe est placée derrière la lame $\mathrm{Mn} \mathrm{Bi}$. Un photomultiplicateur permet l'analyse de l'intensité diffractée par les hologrammes.

4.2. RÉSUltATS EXPÉRIMENTAUX. - Une première série d'inscriptions de réseaux holographiques de différents pas (entre 0,5 et $3 \mu \mathrm{m}$ ) sur des couches minces $\mathrm{MnBi}$ en phase stable nous a permis de définir les conditions optimales pour la formation d'un hologramme magnétique d'une surface voisine de $1 \mathrm{~mm}^{2}$. Les largeurs d'impulsions que nous avons utilisées avec le laser Rubis étaient de $10 \mathrm{~ns}$ et l'énergie nécessaire est voisine de $50 \mathrm{~mJ} / \mathrm{cm}^{2}$. La figure 10 montre l'aspect de la lame $\mathrm{MnBi}$, après l'inscription d'un hologramme,

(*). Environ $1 \mathrm{~m}$. 


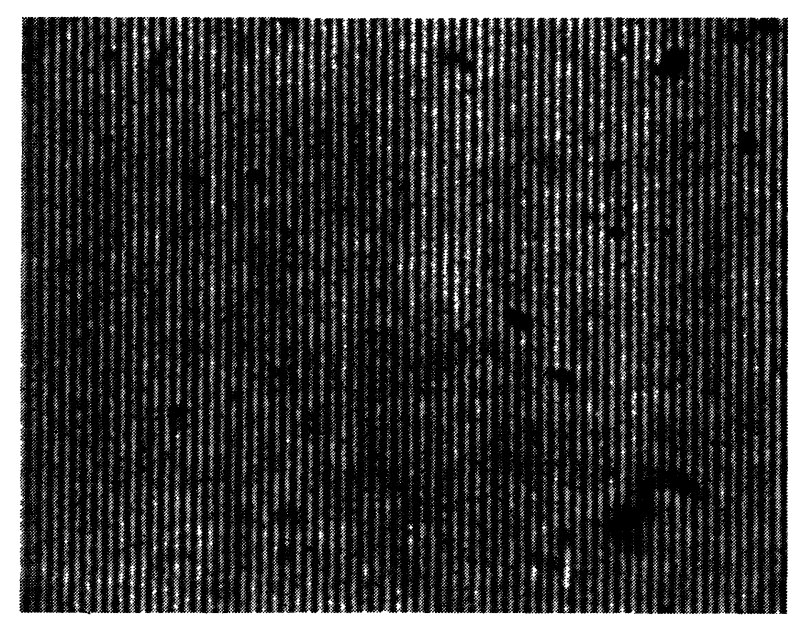

Fig. 10. - Hologramme sur MnBi. Visualisation de la couche au microscope $(G=1000)$ après inscription (interfrange $1,1 \mu \mathrm{m})$.

vu au microscope $(G=1000)$, l'interfrange étant de $1,1 \mu \mathrm{m}$.

De l'intensité diffractée par un réseau inscrit sur $\mathrm{MnBi}$ nous pouvons déduire la répartition d'aimantation obtenue après illumination [10]. Les rapports d'intensités trouvés dans les différents ordres ainsi que la direction de polarisation de ces ordres montrent que le réseau inscrit est proche d'un réseau binaire. En effet, le rapport $I^{\prime \prime} \mid I^{\prime}$ (intensité diffractée dans le $2^{\mathrm{e}}$ ordre par rapport à celle diffractée dans le premier ordre) est compris entre 0,5 et 0,125 . Le cal-

\section{SANS POLAR ISEUR}
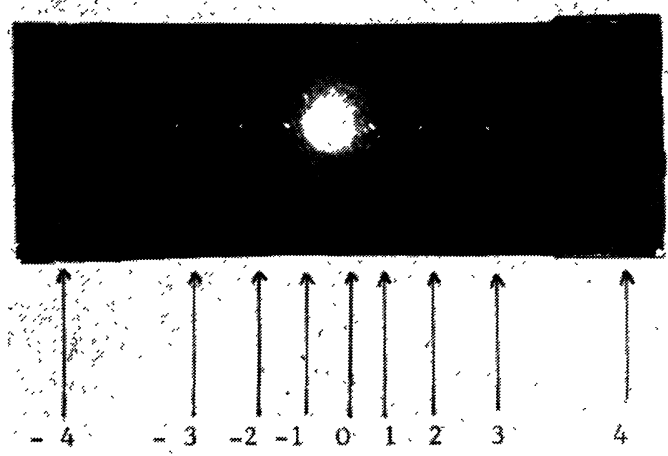

ordrax

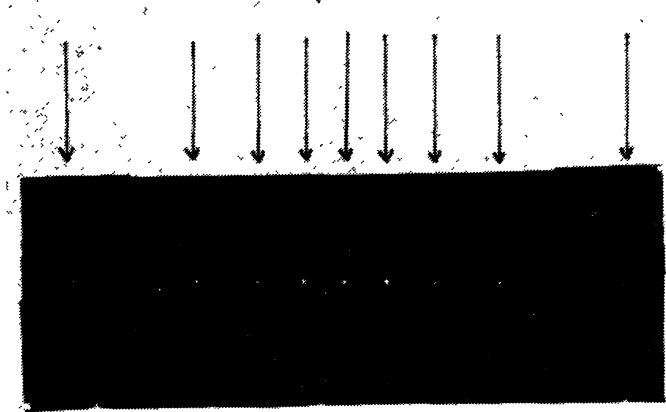

AVEC POLARISEUR CROISE AVEC L'ORDRE O

Fig. 11. - Figure de diffraction d'un réseau holographique sur $\mathrm{MnBi}$. cul théorique pour une répartition symétrique en bande d'aimantation $+M_{s}$ et $-M_{s}$ donne $I^{\prime \prime} \mid I^{\prime}=0,25$. Nous remarquons, comme l'indique la figure 11 que les directions de polarisations des différents ordres diffractés sont croisées avec celle de l'ordre 0 ; les valeurs des intensités relatives de ces ordres sont données sur la figure 12 dans le réseau de pas $1 \mu \mathrm{m}$.

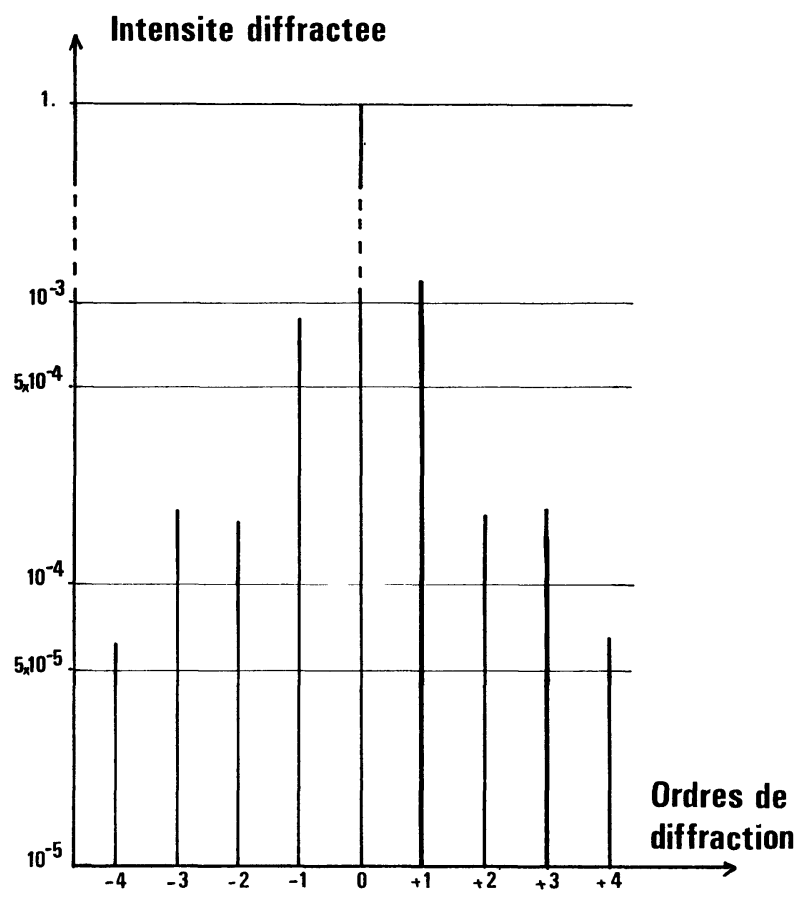

FIG. 12. - Intensité diffractée par un réseau holographique.

L'efficacité des réseaux holographiques inscrits sur $\mathrm{MnBi}$ mesurée par effet Kerr ou Faraday est comprise entre $0,5 \times 10^{-4}$ et $10^{-4}$. Etant donné que nous sommes en présence d'un hologramme mince le rendement de diffraction est constant même si le faisceau de lecture s'écarte d'un angle de $30^{\circ}$ par rapport à la normale à la lame. L'ensemble de ces expé-

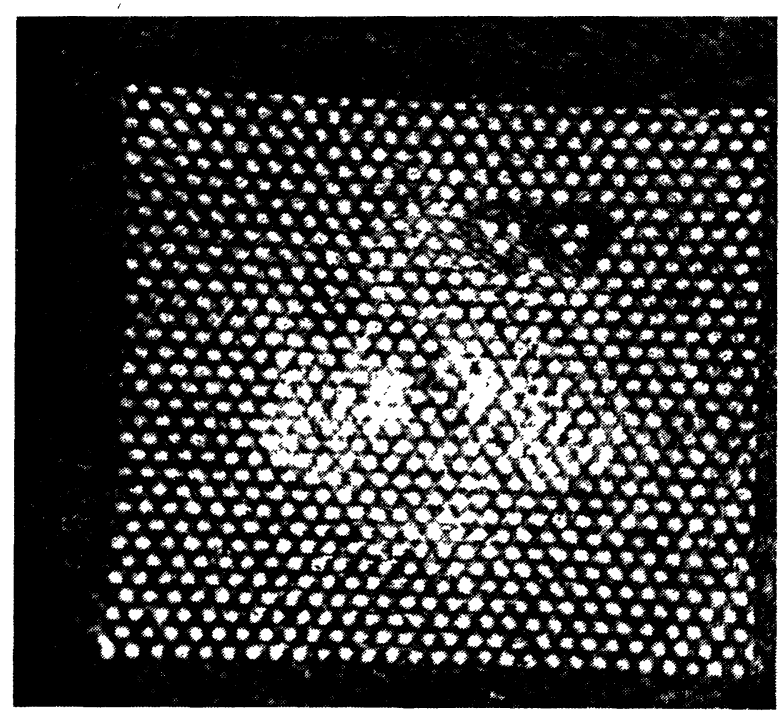

FIG. 13. - Hologramme sur $\mathrm{MnBi}$. Image restituée d'un objet de $30 \times 30$ points. 
riences a permis d'optimiser les conditions d'enregistrement d'hologrammes d'objets binaires. La figure 13 nous montre l'image, restituée en effet Faraday, d'un objet de $20 \times 20 \mathrm{~mm}$ comportant $30 \times 30$ points.

L'effacement sélectif d'un tel hologramme est obtenu en superposant une impulsion de champ à une impulsion lumineuse. L'impulsion de champ permet la réorientation de l'aimantation dans le sens initial de la couche, l'impulsion laser permettant de chauffer au-delà de $T_{\mathrm{c}}$ toute la zone où l'hologramme est inscrit. Les champs utilisés à l'effacement sont voisins de 1200 Oe.

Les techniques holographiques permettent donc d'enregistrer en bloc une page d'informations sur une lame MnBi. L'image restituée est moins sensible aux défauts des couches que dans le cas de l'enregistrement digital, car la répartition spatiale de l'information est plus homogène ; dans les 2 modes d'enregistrement cependant les densités de stockage d'information sont identiques, de l'ordre de $10^{7} \mathrm{bits} / \mathrm{cm}^{2}$.

5. Conclusions. - La présente étude montre dans quelles conditions les lames minces de $\mathrm{MnBi}$ sont utilisables pour le stockage optique d'information avec des densités voisines de $10^{7} \mathrm{bits} / \mathrm{cm}^{2}$.
La phase $\varphi_{\mathrm{m}}$ "à bas point de Curie » présente des caractéristiques plus favorables que la masse $\varphi_{\mathrm{s}}$ à « haut point de Curie » car malgré une aimantation à saturation plus faible elle s'avère plus tolérante quant aux puissances requises à l'enregistrement comme à l'effacement. Toutefois cette phase $\varphi_{m}$ ne présentant pas à température ambiante une stabilité suffisante, il a été nécessaire de remédier à cet inconvénient $[7,8]$ pour que les couches minces de $\mathrm{MnBi}$ puissent jouer un rôle dans le domaine de l'informatique, notamment pour l'enregistrement digital d'informations.

En ce qui concerne l'enregistrement holographique, le même problème de stabilisation de phase se pose. Toutefois, pour ce mode d'enregistrement une limitation pratique provient de la source lumineuse employée. Il est en effet nécessaire d'utiliser un laser haute puissance puisque les temps d'inscription sont de l'ordre de $10 \mathrm{~ns}$ et les énergies requises de $50 \mathrm{~mJ} / \mathrm{cm}^{2}$ pour la phase $\varphi_{\mathrm{s}}, 10$ à $15 \mathrm{~mJ} / \mathrm{cm}^{2}$ pour la phase $\varphi_{\mathrm{m}}$; la fréquence de répétition de tels lasers est encore trop faible pour obtenir des débits suffisants. Cependant, si on peut espérer que dans un proche avenir de tels lasers soient susceptibles de fonctionner à $5 \mathrm{kHz}$, des débits de $100 \mathrm{M}$ Bauds pour une page de $2 \times 10^{4}$ bits pourraient être atteints.

\section{Bibliographie}

[1] Duda, E., Etude du composé $\mathrm{MnBi}$. Thèse $3^{\mathrm{e}}$ cycle. Université Paris Sud (juillet 1971).

[2] Duda, E., Marchal, M., Cizeron, G., C. R. Hebd. Sean. Acad. Sci. 273 (1971) 29.

[3] Duda, E., Marchal, M., A. I. P., Conference proceeding 1971 (5) 1093.

[4] Langlet, R., Pivot, J. P., Carre, B., Revue Technique TH-CSF 4 (1972) 257-281.

[5] Mezrich, R. S., Appl. Opt. 9 (1970) 2275.
[6] Chen, D., Ready, J. F. and Bernal, E., J. Appl. Phys. 39 (1968) 3916.

[7] Aagard, R. L., Schmit, F. M., Lin, T. S. and Di Chen, IE E E Trans. on Magnetics Mag 9 (1973).

[8] Unger, W. K., Wolfgang, E., Harms, H., Haudek H., J. Appl. Phys. 43 (1972) 2875.

[9] Duda, E., Marchal, M., Langlet, R., Proceeding of the International Conference on Magnetism Moscou 1973.

[10] Haimm, Haskal, I E E E Trans. on Mag. Mag 6 (1970) 542. 